\title{
Alternating Field Electronanofluidization
}

Cite as: AIP Conference Proceedings 1145, 97 (2009); https://doi.org/10.1063/1.3180095 Published Online: 01 July 2009

M. J. Espin, J. M. Valverde, M. A. S. Quintanilla, and A. Castellanos

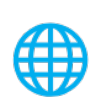

\section{ARTICLES YOU MAY BE INTERESTED IN}

Quasi-static Compaction of Polyhedra by the Discrete Element Method

AIP Conference Proceedings 1145, 90 (2009); https://doi.org/10.1063/1.3180078

The Quantitative Mineralogy of Granular Materials

AIP Conference Proceedings 1145, 87 (2009); https://doi.org/10.1063/1.3180070

\section{Lock-in Amplifiers up to $600 \mathrm{MHz}$}
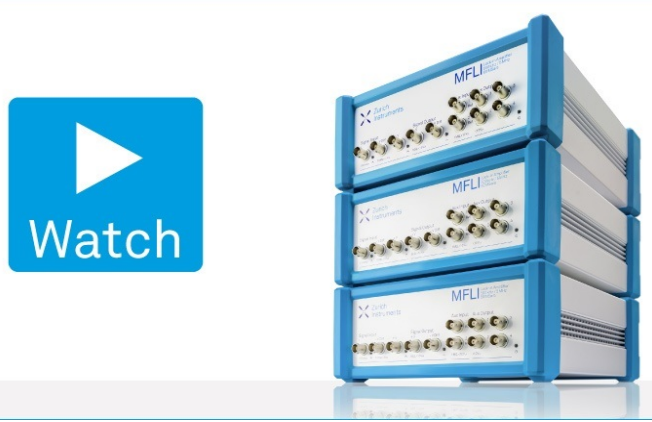


\title{
Alternating Field Electronanofluidization
}

\author{
M. J. Espin*, J. M. Valverde ${ }^{\dagger}$, M. A. S. Quintanilla ${ }^{\dagger}$ and A. Castellanos ${ }^{\dagger}$ \\ ${ }^{*}$ Department of Applied Physics II. University of Seville. Avenida Reina Mercedes s/n, 41012 Seville, Spain. \\ ${ }^{\dagger}$ Faculty of Physics. University of Seville. Avenida Reina Mercedes s/n, 41012 Seville, Spain.
}

\begin{abstract}
The use of fluidized beds to remove submicron particles from gases has been investigated since 1949. High efficiency removal was achieved in the 1970's by imposing an electric field on a fluidized bed of semi-insulating granules that were able to collect the charged pollutant entrained in the fluidizing gas. In spite of their extended use nowadays, the collection efficiency of electrofluidized beds (EFB) is still hindered by gas bypassing associated to gas bubbling and the consequent requirement of too high gas flow and pressure drop. In this paper we report on the electromechanical behavior of an EFB of insulating nanoparticles. When fluidized by gas, these nanoparticles form extremely porous light agglomerates of size of the order of hundreds of microns that allow for a highly expanded nonbubbling fluidized state at reduced gas flow. It is found that fluidization uniformity and bed expansion are additionally enhanced by an imposed AC electric field for field oscillation frequencies of several tens of hertzs and field strengths of the order of $1 \mathrm{kV} / \mathrm{cm}$. For oscillation frequencies of the order of hertzs, or smaller, bed expansion is hindered due to electrophoretic deposition of the agglomerates onto the vessel walls, whereas for oscillation frequencies of the order of kilohertzs, or larger, electrophoresis is nullified and bed expansion is not affected. According to a proposed model, the size of nanoparticle agglomerates stems from the balance between shear, which depends on field strength, and van der Waals forces. The optimum field strength for enhancing bed expansion produces an electric force on the agglomerates similar to their weight force, while the oscillation velocity of the agglomerates is similar to the gas velocity.
\end{abstract}

Keywords: Fluidized beds, Nanopowders, Filters

PACS: 47.55.Lm, 81.07.Wx, 84.30.Vn

\section{INTRODUCTION}

Efficient removal of dust and mist from gases by passing these through a bed of fluidized solids was first claimed by Meisner and Mickley in 1949 [1]. Almost two decades later, practical problems such as reducing air pollution from diesel exhaust and filtration of smoke emissions from asphaltic pavement recycling process, motivated Melcher to develop electrofluidized beds for collection of submicron particles [2]. Melcher and coworkers stressed a fluidized bed by imposing an electric field that effectively polarized the millimeter sized particles. These semi-insulating particles then served the function of conventional electrostatic precipitators, acting as collection sites for the charged pollutants entrained in the fluidizing gas. In comparison to electrostatic precipitators, the collection surface area per unit volume of electrofluidized beds is greatly increased, making it possible to reduce the gas residence time and volume of the filter [2]. A major concern that besets classical fluidized bed filters lies in the bypassing of the contaminants through gas bubbles. Gas bubbling is the typical behavior found in fluidized beds of millimeter sized particles [3], providing little gas-solid contact and hampering reaction efficiency.

Powders of moderate density nanoparticles (such as silica) can be uniformly fluidized and experience a transition to elutriation at high gas velocities with full sup- pression of visible bubbles [4]. In this paper we study the behavior of an electrofluidized bed of dry silica nanoparticles, which shows agglomerate particulate fluidization in the absence of externally applied electric field. Samples were subjected to DC and AC electric fields in order to check the influence of the frequency of the external field and to determine the size and charge of the powder. The suppression of gas bubbles in fluidized beds of nanoparticles has been causally related to the formation of porous light agglomerates. A phenomenological approach to predict the behavior of gas-fluidized beds of nanoparticles is to consider agglomerates as effective low-density spheres which may exhibit nonbubbling gasfluidization similarly to coarse particles fluidized by liquids. In this way, the Richardson-Zaki (RZ) equation, originally intended to describe the expansion of uniform liquid-fluidized beds [6], can be modified to consider uniform gas-fluidization of nanoparticle agglomerates [4]

$$
\frac{v_{g}}{v_{p}}=k_{a}^{D-1}\left(1-k_{a}^{3-D} \phi\right)^{n}
$$

where $v_{g}$ is the superficial gas velocity, $v_{p}$ is the terminal settling velocity of a single particle, $\phi$ is the particle volume fraction, $k_{a}=d^{* *} / d_{p}$ is the ratio of agglomerate size to particle size, and $D=\ln N_{a} / k_{a}$, where $N_{a}$ is the number of particles in the agglomerate. 


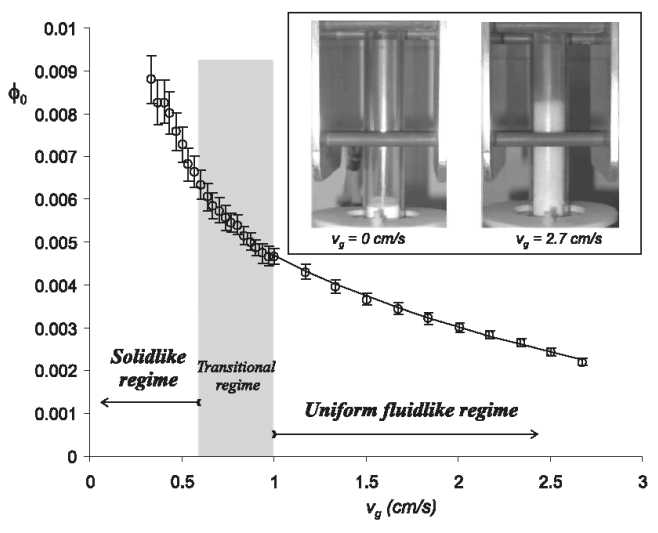

FIGURE 1. Particle volume fraction as a function of superficial gas velocity for fluidization without external electric field applied. The solid line represents the modified RZ equation fit to the data in the uniform fluidlike regime. The photographs in the inset illustrate bed expansion.

\section{EXPERIMENTAL SETUP AND RESULTS}

The fluidized bed apparatus consisted of a vertical 2.54 $\mathrm{cm}$ internal dia. polycarbonate vessel, $16.2 \mathrm{~cm}$ in height, which is fitted at the bottom with a sintered stainless steel plate, having a pore size of $5 \mu \mathrm{m}$, that acts as gas distributor. The fluidization cell is is placed between two parallel square electrodes of $14 \mathrm{~cm}$ of side length. The distance between the electrodes is fixed to $l=8 \mathrm{~cm}$. One of the electrodes is grounded and high voltage $V$ is applied to the opposite electrode from an oscillator/amplifier network. The electric field strength within the bed can be taken as approximately constant $(E \simeq V / l)$. The power supply system allowed us to provide peak field strengths up to $E_{0} \simeq 2 \mathrm{kV} / \mathrm{cm}$ over a frequency range up to $10 \mathrm{kHz}$. Different types of waveform, sinusoidal, square and triangular shape, were applied. The flow of gas (dry air) to the column was controlled by means of a MKS flow controller with a full range from 0 to $2000 \mathrm{~cm}^{3} / \mathrm{min}$. The material tested in the experiments was pre-sieved (using a sieve opening of $500 \mu \mathrm{m}$ ) Aerosil(CR974, which is a hydrophobic $\mathrm{SiO}_{2}$ nanopowder with a particle density of $\rho_{p}=2250 \mathrm{~kg} / \mathrm{m}^{3}$, and particle size $d_{p}=12 \mathrm{~nm}$.

In Figure 1 we have plotted experimental data of $\phi$ as a function of $v_{g}$ in the absence of external field applied. The bed expands monotonously as $v_{g}$ is increased. By fitting the data of $v_{g}$ vs. $\phi$ to the modified RichardsonZaki equation (Eq. 1), it is obtained $d^{* *}=226 \mu \mathrm{m}$ and $D=2.588$, which is in good agreement with previous measurements [4].

Figure 2 shows experimental data on $\phi$ as a function of $v_{g}$ and for different values of the strength of the crossflow electrostatic field applied. The main effect of the electric field is an increase of $\phi$. From local observations [7], it is seen that the dynamics is ruled by elec-

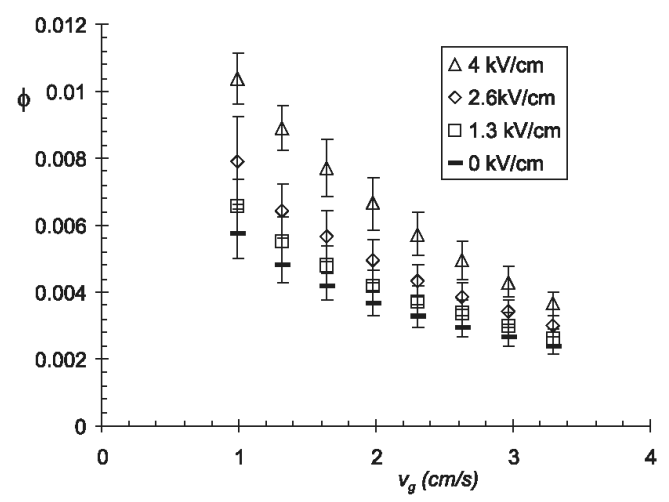

FIGURE 2. . Average particle volume fraction of the fluidized bed $\phi$ as a function of the superficial gas velocity $v_{g}$ for different strengths of the electrostatic field applied.

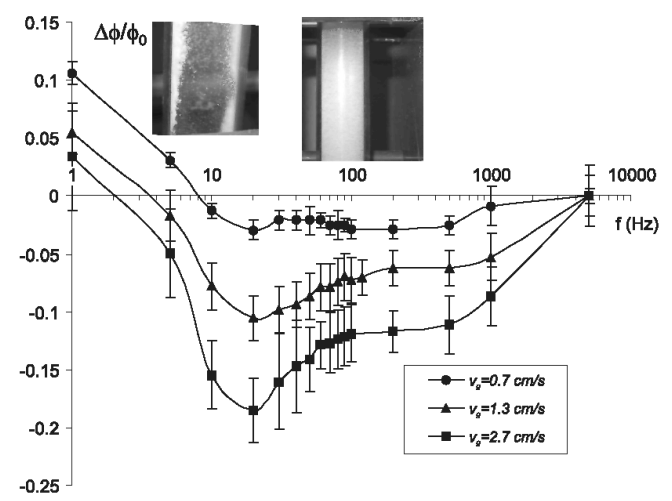

FIGURE 3. Relative variation of the particle volume fraction as a function of the oscillation frequency of the alternating electric field. Peak field strength is fixed to $1.25 \mathrm{kV} / \mathrm{cm}$. Data is shown for three different values of the superficial gas velocity $v_{g}$. The inset shows electrophoretic deposition $(1 \mathrm{~Hz})$ and enhanced bed expansion $(20 \mathrm{~Hz})$.

trophoretic deposition at the wall. In the stationary state, fluidization quality is hampered and bed expansion is decreased.

Insulating dry particles in a fluidized bed accumulate a significant amount of charge. Most investigations suggest that the main cause for the charge build-up on granular materials during industrial handling and processing is contact charging [5]. Charges are exchanged whenever any two surfaces come into contact with each other even for cases where the bulk materials are the same due to surface impurities and imperfections. The electrophoretic force on our agglomerates is $F_{e} \simeq Q^{* *} E$, where $Q^{* *}$ is their charge. Using the Stokes' law for an isolated agglomerate, it can be estimated $Q^{* *}=$ $3 \pi \mu d^{* *} v_{h}^{* *} / E$, where $\mu$ is the gas viscosity and $v_{h}^{* *}$ is the terminal velocity of the agglomerate. We have analyzed the trajectories of some agglomerates at the initial stage of application of the electric field by means of a 

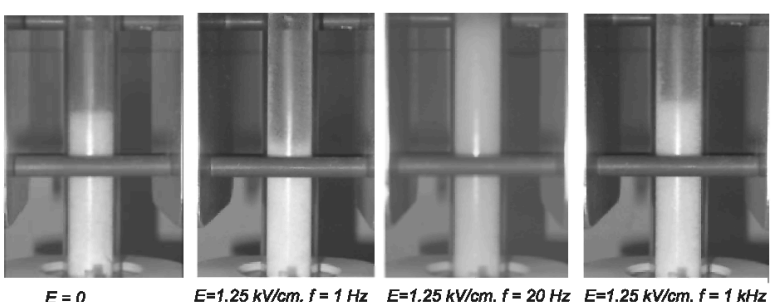

FIGURE 4. Photographs of the electronanofluidized bed illustrating bed expansion for three different frequencies of the alternating electric field (indicated) as compared to bed expansion in the absence of externally applied electric field. Superficial gas velocity is fixed to $v_{g}=2.7 \mathrm{~cm} / \mathrm{s}$.
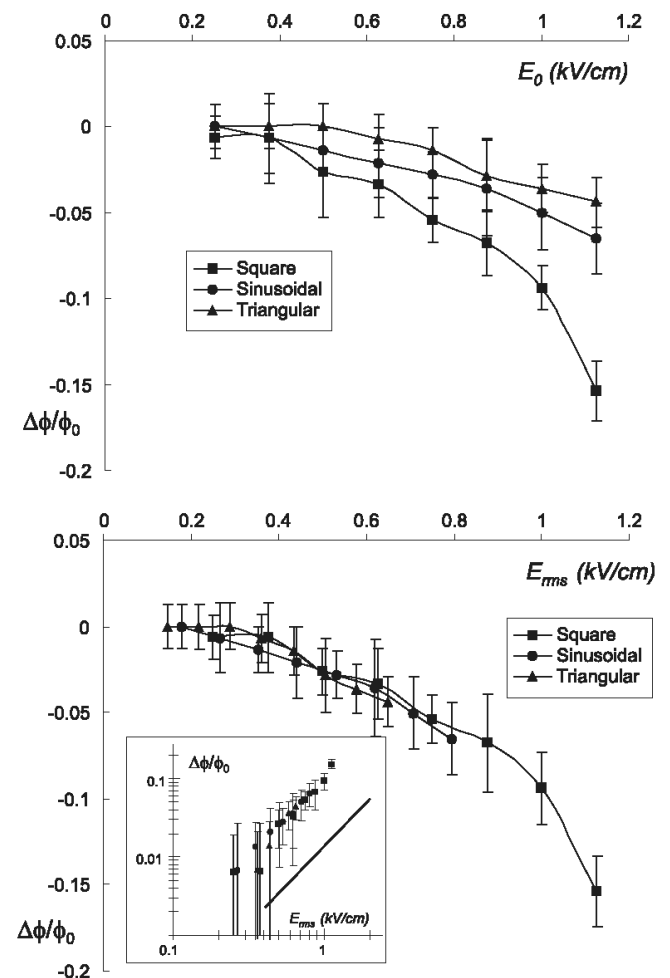

FIGURE 5. Relative variation of the particle volume fraction of the electronanofluidized bed as a function of the field strength for three different waveform types. Top: Data is shown as a function of the peak field strength $E_{0}$. Bottom: Data is shown as a function of the root-mean-squared field strength $E_{r m s}$. electric field oscillation frequency and superficial gas velocity are fixed to $20 \mathrm{~Hz}$ and $v_{g}=2.7 \mathrm{~cm} / \mathrm{s}$, respectively.

CMOS high-speed camera. The agglomerate charge of the agglomerates manually tracked was found to be of the order of $10^{-14} \mathrm{C}$, giving a charge to mass ratio $\mathrm{Qmr}$ in the range $10^{-5}-10^{-4} \mathrm{C} / \mathrm{kg}$.

In the case of an alternating field, it is expected that the charged agglomerates will be forced to oscillate at the field frequency. Figure 3 shows the relative variation

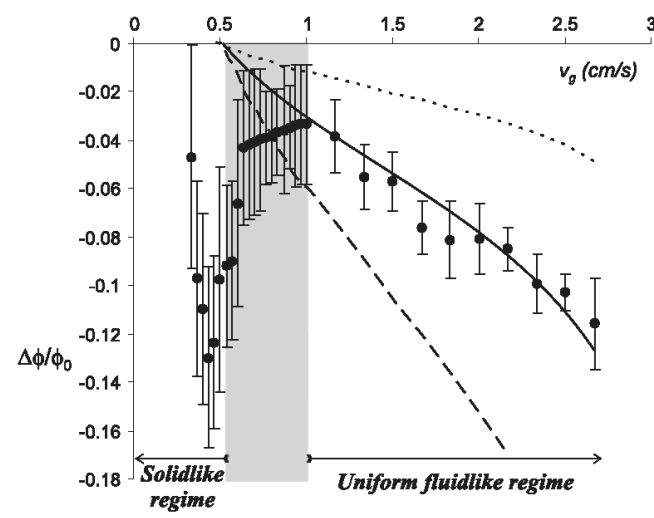

FIGURE 6. Relative variation of the particle volume fraction of the electronanofluidized bed as a function of the superficial gas velocity. Electric field oscillation frequency and strength are fixed to $500 \mathrm{~Hz}$ and $E_{0}=1.25 \mathrm{kV} / \mathrm{m}$ (square wave), respectively. The lines are predicted curves by the model. Solid line: complex-agglomerate charge $Q_{0}^{* *}=1.9 \times 10^{-14} \mathrm{C}$. Dotted line: $Q_{0}^{* *}=1 \times 10^{-14} \mathrm{C}$. Dashed line: $Q_{0}^{* *}=3 \times 10^{-14} \mathrm{C}$

of the particle volume fraction $\left(\Delta \phi / \phi_{0}\right.$, where $\phi_{o}$ is the particle volume fraction in the absence of external electric field) of the electronanofluidized bed as a function of the oscillation frequency for a fixed peak field strength $\left(E_{0}=1.25 \mathrm{kV} / \mathrm{cm}\right)$. For frequencies of the order of hertzs the main mechanism is still electrophoretic deposition of the agglomerates on the walls, which gives rise to bed channeling and collapse. In the range of intermediate frequencies, between tens and hundreds of hertzs, the opposite behavior is observed. Bed expansion is greatly enhanced by the alternating field. Finally, for frequencies of the order of kilohertzs and larger, the alternating field has no appreciable effect on bed expansion (see Fig. 4). The effect of the strength of the field in the range of intermediate oscillation frequencies and waveform type is shown in Fig. 5. Bed expansion is further enhanced as the strength of the field is increased. The data matches to a single trend when it is plotted against the root-meansquared field $E_{r m s}$, which indicates that enhanced bed expansion is the result of a time averaged process. From a $\log -\log$ plot (see inset) it is observed that $\Delta \phi / \phi_{0}$ scales as the field strength squared, which is proportional to the kinetic energy gained by the agglomerates during one semiperiod. This suggests a possible role of collisions between agglomerates of different charge on deagglomeration.

\section{DISCUSSION}

A change of the particle volume fraction when the field is turned on could be attributable to a variation of the complex-agglomerate size. In order to estimate 
theoretically the complex-agglomerate size, simpleagglomerates, which exist before fluidization [4], will be considered as effective particles undergoing agglomeration due to attractive forces between each other [8]. This attractive force is counterbalanced by the gas flow shear to support the weight of the complex-agglomerate in the gravity field plus the shear due to their forced oscillations in the electric field. The balance between the overall time-averaged shear and the attractive force determines the size of the complex-agglomerates. The complex-agglomerate weight force $W^{* *}$ is balanced by the hydrodynamic friction from the surrounding gas, which acts mainly at its surface due to the flow screening effect. Using a spring model [8], the typical strain on the surface of the complex-agglomerate can be estimated as $\gamma_{g} \sim W^{* *} / K^{* *} R^{* *}$. Here $W^{* *}=N^{*} W^{*}$, where $N^{*}$ is the number of simple-agglomerates in the complex-agglomerate and $W^{*}$ is the simple-agglomerate weight force. $K^{* *}$ is the complex-agglomerate spring constant, which, using a theory on the elastic properties of random percolating systems, can be obtained from the simple-agglomerate spring constant $K^{*}$ and the elasticity exponent $\beta$ ( $K^{* *} \sim K^{*} /\left(k^{*}\right)^{\beta}$, where $k^{*}$ is the ratio of complex-agglomerate radius $R^{* *}$ to simple-agglomerate radius $R^{*}$ and $\beta=3$ in 3D). When the alternating electric field is turned on, the forced complex-agglomerate oscillations give rise to an additional shear force to balance the electric force $Q^{* *} E$. The root-mean-squared shear strain is thus increased up to $\gamma_{m s}=\sqrt{\left(\gamma_{g}\right)^{2}+\left(\gamma_{E}\right)^{2}}$, where $\gamma_{E} \sim Q^{* *} E_{r m s} / K^{* *} R^{* *}$. Thus, the shear force, which acts on the simple-agglomerates attached to the outer layer of the complex-agglomerate, is $F_{s} \sim K^{*} \gamma_{r m s} R^{*} \sim\left(k^{*}\right)^{D+2} \sqrt{\left(W^{*}\right)^{2}+\left(Q^{*} E_{r m s}\right)^{2}}$. Here it is assumed $N^{*}=\left(k^{*}\right)^{D}$ and that the complexagglomerate charge is equally distributed among the simple-agglomerates $\left(Q^{* *}=N^{*} Q^{*}\right.$, where $Q^{*}$ is the simple-agglomerate charge). Simple-agglomerates attach to the complex-agglomerate as long as the shear force is smaller than the attractive force. Thus, the limit condition $F_{s}=F_{v d W}$ leads to the equation $k^{*} \sim B o^{1 /(D+2)}$ for estimating the complex-agglomerate size $d^{* *}$, where the agglomerate Bond number $B o$ is defined as the ratio of the attractive force $F_{v d W}$ to $\sqrt{\left(W^{*}\right)^{2}+\left(Q^{*} E_{r m s}\right)^{2}}$. The simple-agglomerate weight is $W^{*}=N_{p} W_{p}$, where $W_{p}$ is the nanoparticle weight and $N_{p}$ is the number of primary nanoparticles in the simpleagglomerate, which is assumed to be $N_{p}=\left(d^{*} / d_{p}\right)^{D}$. The simple-agglomerate charge $Q^{*}$ is needed to estimate the complex-agglomerate size. Provided that simpleagglomerates are unaltered by the electric field, it is $Q^{*}=Q_{0}^{* *} / N_{0}^{*}$, where $Q_{0}^{* *}$ and $N_{0}^{*}$ are the complexagglomerate charge and number of simple-agglomerates in the complex-agglomerate for $E=0$, respectively. Thus

$$
k^{*}=k_{0}^{*}\left(1+\left(\frac{Q_{0}^{* *} E_{r m s}}{\left(k_{0}^{*}\right)^{D}}\right)^{2}\right)^{-1 /(2(D+2))}
$$

where $k_{0}^{*}=d_{0}^{* *} / d^{*}$ is the relative complex-agglomerate size in the absence of electric field. Using a typical value of $F_{v d W}=10 \mathrm{nN}, d^{*}=30 \mu \mathrm{m}$, and $D=2.5-2.6$ [4], it is predicted $d_{0}^{* *} \simeq 150 \mu \mathrm{m}$ in the absence of applied electric field. Eq. 2 can be used in the modified RZ equation (Eq. 1). Figure 6 shows the relative variation of the particle volume fraction as a function of the superficial gas velocity for an imposed external field of peak strength $E_{0}=1.25 \mathrm{kV} / \mathrm{cm}$. The lines represent the predictions by the model for different values of the complexagglomerate charge. Complex-agglomerate size in the absence of electric field and fractal dimension are taken from our previous derivation based on bed expansion experimental data $\left(d_{0}^{* *}=226 \mu \mathrm{m}, D=2.588\right.$ ). Remarkably, the prediction fits to the data in the fluidlike regime for a value of the complex-agglomerate charge $\left(Q_{0}^{* *}=\right.$ $1.9 \times 10^{-14} \mathrm{C}$ ) similar to the order of magnitude estimated by tracking agglomerate trajectories. The model prediction is however quite sensitive to the agglomerate charge. Considerable deviation from the data is obtained for other charge values of same order of magnitude. This prevents us from a conclusive statement on the model validity. Moreover, the role of collisions on deagglomeration is not considered by the model.

\section{ACKNOWLEDGMENTS}

We acknowledge Spanish Government Agency Ministerio de Ciencia y Tecnologia (contract FIS2006-03645) and Junta de Andalucia (contract FQM 421).

\section{REFERENCES}

1. H. P. Meissner, H. S. Mickley, Ind. Eng. Chem. 47 (1949) $1238-1242$.

2. J R. Melcher, Proceedings of the IEEE 65 (1977) 1659-1672.

3. D. Geldart, Powder Technol. 7 (1973) 285-292.

4. J. M. Valverde, A. Castellanos, Chem. Eng. Sci. 62 (2007) 6947-6956.

5. M. Glor, John Wiley \& Sons, New York, 1988.

6. J.F. Richardson, W.N. Zaki, Trans. Inst. Chem. Engrs. 32 (1954) 35-53.

7. J. M. Valverde, M.A.S. Quintanilla, M.J. Espin, A. Castellanos, Phys. Rev. E 77 (2008) 031301.

8. A. Castellanos, J.M. Valverde, M.A.S. Quintanilla, Phys. Rev. Lett. 94 (2005) 075501(1)-075501(4). 